\title{
Multi-layered Graphene Based Optically Tunable Terahertz Absorber
}

\author{
Alexander N. Grebenchukov ${ }^{1,2}$, Mikhail G. Novoselov ${ }^{1}$, Anton D. Zaitsev ${ }^{1}$, Evgeniya O. Kovalska ${ }^{3}$, \\ Anna V. Baldycheva ${ }^{3}$, and Mikhail K. Khodzitsky ${ }^{1}$ \\ ${ }^{1}$ Terahertz Biomedicine Laboratory, ITMO University, Saint Petersburg, 199034 Russia \\ ${ }^{2}$ Opto-Electronics and Measurements Techniques Laboratory, University of Oulu, 90014, Finland \\ ${ }^{3}$ Centre for Graphene Science, University of Exeter, Exeter EX4 4QL, UK
}

\begin{abstract}
THz})$ absorber, which consists of cross-shaped multi-layered graphene (MLG) resonators. The proposed absorber possesses almost perfect absorption over the range 0.4-0.8 $\mathrm{THz}$ and can be easily fabricated. Dynamical tuning of absorption band is achieved by external optical pumping of modest intensity. This multi-layered graphene-based absorber has high potential for various $\mathrm{THz}$ applications
\end{abstract}

\section{INTRODUCTION}

$\mathrm{G}$ RAPHENE metamaterial devices particularly absorbers have become recently in the focus of research attention, especially in the THz frequency range [1-4]. In comparison with conventional $\mathrm{THz}$ absorbers, graphene-based ones possess tuning capability of absorption characteristics in terms of resonant frequency and absorption value due to the graphene unique and adjustable electronic transport properties. Moreover, graphene can interact with radiation in millimeter, infrared and visible range of wavelengths, so using this material the possibility of $\mathrm{THz}$ radiation control by optical pumping can be achieved. However, most of existing graphene $\mathrm{THz}$ absorbers usually have low and narrowband absorption in the $\mathrm{THz}$ frequency range due to the limits concerning weak light-matter interaction in graphene monolayer and its resonant nature. To realize high wideband absorption, multi-resonance and multi-layering approaches are utilized, which are difficult in practical implementation [5]. Therefore, development of a tunable near-perfect broadband $\mathrm{THz}$ absorber which can be easily manufactured is an important task.

In this work, we propose an optically tunable wideband $\mathrm{THz}$ absorber based on multi-layered structured graphene (about 80 layers) that achieves almost perfect absorption over $0.4-0.8 \mathrm{THz}$ and can be easily fabricated.

\section{RESULTS}

The designed unit cell of multi-layered graphene-based absorber metasurface is shown in Fig. 1. The structure consists of cross-shaped MLG resonators, $0.5 \mu \mathrm{m}$ thick aluminum bottom film (with conductivity $\sigma=3.56 \times 10^{7} \mathrm{~S} / \mathrm{m}$ ) and $80 \mu \mathrm{m}$ thick dielectric (with permittivity $\varepsilon=2.1$ ) spacer between them (made from TPX material which is near-fully transparent in the $\mathrm{THz}$ frequency range). The boundary conditions are selected to be periodical along $\mathrm{x}$ - and $\mathrm{y}$-axis, and the $\mathrm{THz}$ radiation propagates along $\mathrm{z}$-axis from the graphene-coated side. $\mathrm{THz}$ wave reflects from the aluminum film, so it interacts with graphene two times: it increases the efficiency of the device and makes it work in the reflection (absorption) mode. The parameters of the device are controlled by applying continuous-wave (CW) external optical pumping with wavelength of $980 \mathrm{~nm}$ and intensity in range from 0 to 10 $\mathrm{mW} / \mathrm{mm}^{2}$, which significantly changes the $\mathrm{THz}$ complex conductivity of multi-layered graphene.

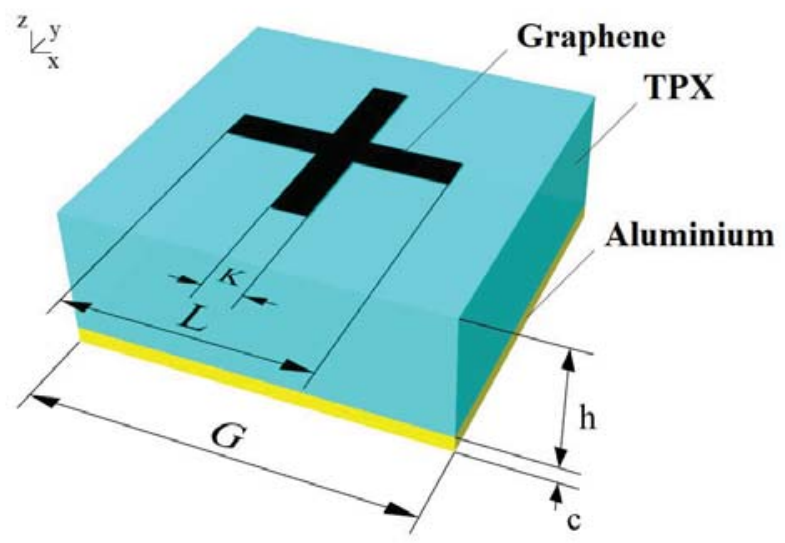

Fig. 1. Unit cell geometry of the proposed optically tunable absorber based on multi-layered graphene metasurface. The geometrical dimensions are the next: $K=20 \mu \mathrm{m}, L=175 \mu \mathrm{m}, G=200 \mu \mathrm{m}, h=80 \mu \mathrm{m}$, and $c=0.5 \mu \mathrm{m}$.

To obtain absorption characteristics of the proposed $\mathrm{THz}$ device (Fig. 3), we performed numerical simulations based on finite element method (FEM) using CST Microwave Studio suite. The MLG was defined as infinitely thin film with experimentally obtained conductivity spectra in the $0.3-1.0$ $\mathrm{THz}$ frequency range (Fig. 2) [6]. The MLG sample was fabricated by chemical vapor deposition (CVD) method in quartz furnace. The optoelectronic properties of this sample were studied by pulsed terahertz time-domain spectroscopy (THz-TDS) method in transmission mode using thin-film approximation (when the wavelength of $\mathrm{THz}$ radiation is much more than thickness of MLG) for the different infrared optical pumping intensities. The measurements were performed at room temperature under normal conditions.

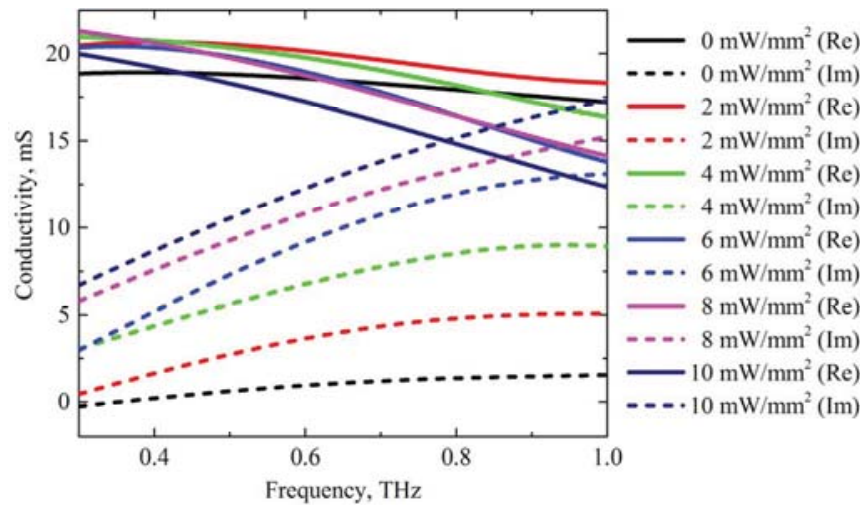

Fig. 2. Real and imaginary part of MLG conductivity vs frequency under optical pumping $(980 \mathrm{~nm})$ of different intensities. 


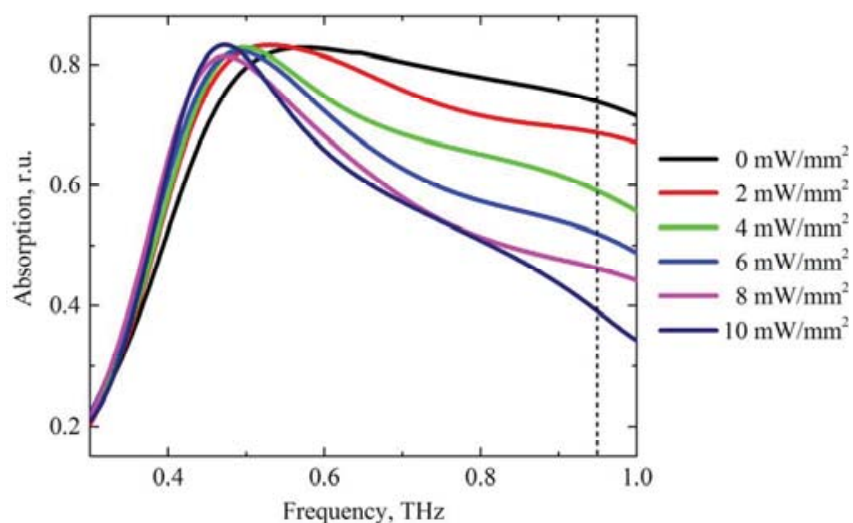

Fig. 3. Absorption spectra of MLG-based $\mathrm{THz}$ absorber under different optical pumping intensities.

As it can be seen from Fig. 2, the external optical pumping significantly changes the imaginary part of complex conductivity of MLG. At the same time, there is a tendency to saturation of changes in the spectrum of imaginary part of conductivity. The real part of the complex conductivity varies in a limited range of values.

The absorption spectra of designed device are shown in Fig. 3. As depicted in this figure, there is a significant influence of external optical pumping $(980 \mathrm{~nm})$ of modest intensity over absorption spectrum from $0.3 \mathrm{THz}$ to $1 \mathrm{THz}$. Vertical dashed line in Fig. 3 represents the $0.95 \mathrm{THz}$ frequency point, for which the dependency of absorption coefficient on optical pumping intensity is shown in Fig. 4.

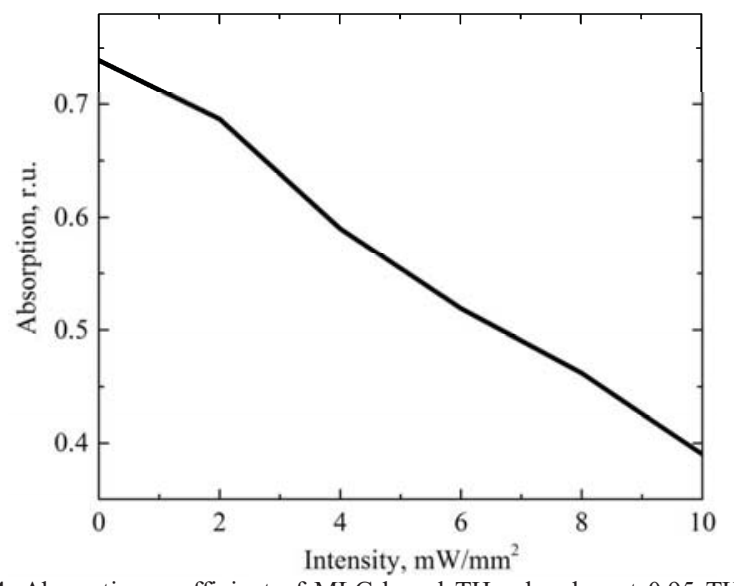

Fig. 4. Absorption coefficient of MLG-based THz absorber at $0.95 \mathrm{THz}$ vs optical pumping intensity.

Fig. 3 shows that the proposed structure works in broadband mode, and the degree of tunability depends on the frequency of $\mathrm{THz}$ radiation. At $0.5-1.0 \mathrm{THz}$, as the intensity of the optical pumping increases, the absorption of the structure decreases. The greatest amplitude of absorption tuning is observed at frequencies near 0.9-1.0 THz. In Fig. 4 it is shown that at $0.95 \mathrm{THz}$ the absorption coefficient of MLG-based absorber near-linearly depends on the intensity of infrared optical pumping and varies from 0.74 to 0.39 r.u. It also should be mentioned that at some frequency points $(0.3$ and $0.5 \mathrm{THz}$ ) the absorption coefficient almost does not depend on the pumping intensity. The position of such frequency points depends on the geometry of the metasurface unit cell.

\section{SUMMARY}

In summary, we have designed and numerically simulated an optically tunable broadband near-perfect $\mathrm{THz}$ absorber based on multi-layered graphene metasurface. Despite the ordered structure of the surface, this device operates in broadband mode. The ultrafast electronic properties of graphene allow to realize high-speed modulation of amplitude of $\mathrm{THz}$ wave which interacts with absorber. The usage of optical pumping method avoids the application of an electrode system and makes the absorber to work in all-optical mode. Moreover, the usage of optical pumping allows to achieve the working frequency of the device, unattainable in the electronic control circuit. Graphene is resistant to atmospheric influences and easily can be manufactured. It was shown that the efficiency of the absorption modulation depends on the frequency of $\mathrm{THz}$ radiation, while the absorption spectra of the structure are determined by its geometric parameters. Relatively weak optical pumping source can be used to control the absorber state. The proposed absorber can be used in many promising $\mathrm{THz}$ applications such as biosensing, imaging and wireless communications (in transparency windows of the atmosphere).

\section{ACKNOWLEDGMENTS}

This work was financially supported by Government of Russian Federation, Grant 08-08.

\section{REFERENCES}

[1]. Su, Zengping, et al., "A tunable THz absorber consisting of an elliptical graphene disk array," Physical Chemistry Chemical Physics, vol. 20, pp. 14357-14361, 2018.

[2]. Xiong, Han, et al., "Ultra-thin and broadband tunable metamaterial graphene absorber," Optics Express, vol. 26, pp. 1681-1688, 2018.

[3] A.N. Grebenchukov, S.E. Azbite, A.D. Zaitsev, and M.K. Khodzitsky "Faraday effect control in graphene-dielectric structure by optical pumping," Journal of Magnetism and Magnetic Materials 472, pp. 25-28, 2019.

[4] A. Grebenchukov, A. Zaitsev, M. Khodzitsky "Optically controlled narrowband terahertz switcher based on graphene," Chinese Optics 11(2), pp. $166-173,2018$

[5]. Deng X. H., et al., "Tunable THz absorption in graphene-based heterostructures," Optics Express, vol. 22, pp. 30177-30183, 2014.

[6]. Grebenchukov, A.N., et al., "Terahertz conductivity of photoexcited multi-layer graphene" 43rd IRMMW-THz, pp. 1-2, 2018 